\title{
Evidências de Validade de uma Escala Destinada à Avaliação da Metacognição Infantil $^{1}$
}

\author{
Jussara Fátima Pascualon-Araujo ${ }^{2}$ \\ Patrícia Waltz Schelini \\ Universidade Federal de São Carlos
}

\begin{abstract}
RESUMO - Pensamentos e conhecimentos que os indivíduos possuem sobre seus próprios processos cognitivos definem o termo metacognição. O objetivo deste trabalho foi continuar o processo de validação da Escala de Metacognição (EMETA) analisando evidências baseadas na estrutura e na consistência internas, bem como a realização de comparação entre as médias para investigar as influências das variáveis gênero, tipo de escola e idade. Os resultados encontrados em amostra de 196 participantes entre 9 e 12 anos apontaram a presença de um único fator denominado Metacognição e a possibilidade de redução dos itens de 67 para 40. Não foi encontrada diferença significativa influenciada pelas variáveis analisadas. Apesar dos resultados favoráveis ao acúmulo das evidências de validade, outros parâmetros psicométricos ainda devem ser avaliados.
\end{abstract}

Palavras-chave: metacognição infantil, avaliação, psicometria

\section{Validity Evidence of a Scale Designed to Assess Children's Metacognition}

\begin{abstract}
Thoughts and knowledge that individuals have about their own cognitive processes define the term metacognition. The aim of this work was to continue the validation process of Metacognition Scale (EMETA) analyzing evidence based on the structure and consistency internal and a comparison between the means to investigate the influences of variables such as gender, school type and age of participants. The results found with 196 participants between 9 and 12 years old indicate the presence of a single factor called Metacognition and the possibility to reduction for 40 items. There was no significant difference influenced by the three variables. Despite the favorable results to the accumulation of evidence of validity, other parameters must still be evaluated on future researches.
\end{abstract}

Keywords: metacognition of children, assessment, psychometrics

Os primeiros relatos sobre o termo metacognição podem ser atribuídos a Flavell $(1976,1979)$ que o definiu como o "conhecimento e cognição sobre o fenômeno cognitivo" $(1979$, p. 906) ou ainda o conhecimento que se tem sobre os próprios processos cognitivos e seus produtos (1976). Dessa maneira, quando o aluno percebe que se desempenha melhor em provas nas quais estuda o conteúdo anotando palavras-chave sobre cada ponto da matéria está utilizando suas estratégias metacognitivas, já que o uso e a compreensão sobre as anotações só são possíveis por meio do conhecimento sobre os próprios processos cognitivos.

Apesar de Flavell apresentar sua definição sobre metacognição apenas na segunda metade da década de 1970, o autor já havia realizado uma pesquisa de cunho metacognitivo com pré-escolares e escolares antes desse período. Flavell, Friedrichs e Hoyt (1970) investigaram a habilidade de crianças entre 5 e 7 anos de idade de recordar itens apresentados anteriormente a elas. Os resultados demonstraram que alguns alunos, especialmente os mais novos, disseram estar prontos para falar todos os itens quando não o estavam, uma vez que, não conseguiram recontar os itens de maneira eficiente. Os autores inferiram que isso se deu devido à incapacidade desses alunos menores de monitorar seus processos cognitivos.

1 Apoio: CAPES

2 Endereço para correspondência: Avenida Leonardo da Vinci, 211, apto 138, São Paulo, Brasil. CEP: 04313-000. E-mail: jussara.pascualon@gmail.com
A importância do termo metacognição, especialmente no que diz respeito ao desenvolvimento cognitivo, originou expressivo interesse e a publicação, em 1979, do artigo de Flavell, considerado um clássico da área (Dunlosky \& Metcalfe, 2009). Nesse artigo, Flavell apresenta o Modelo do Monitoramento Cognitivo com o intuito de explicar o conceito e especificar os domínios da metacognição. De acordo com esse modelo, o monitoramento dos processos cognitivos ocorre por meio de ações entre quatro classes de fenômenos: a) conhecimento metacognitivo; b) experiências metacognitivas; c) objetivos cognitivos e d) estratégias cognitivas. O conhecimento metacognitivo é definido como o conhecimento ou a crença que o indivíduo possui sobre quais fatores interagem e afetam o desempenho cognitivo. É constituído por três variáveis: pessoa, tarefa e estratégia que estão relacionadas às diferenças de desempenho cognitivo entre os indivíduos; à natureza das informações apresentadas pela tarefa em questão e; às informações sobre as formas de alcançar os objetivos propostos pela tarefa de maneira eficaz, respectivamente (Flavell, 1979). As experiências metacognitivas são impressões ou percepções do indivíduo em relação aos eventos de natureza cognitiva ou afetiva, de longa ou curta duração e que podem ocorrer antes, durante ou após a realização de uma tarefa. Já os objetivos cognitivos correspondem ao que se deseja produzir ao término da tarefa, a meta a ser atingida. Por fim, as estratégias cognitivas são os meios utilizados para alcançar os objetivos cognitivos (Flavell, 1979). 
Desde sua criação até o momento, o Modelo do Monitoramento Metacognitivo (Flavell, 1979) vem ganhando novos conceitos advindos da Psicologia Cognitiva, especialmente, por meio da abordagem do Processamento da Informação (Jou \& Sperb, 2006). Contribuição importante foi apresentada por Nelson e Narrens $(1990,1996)$ que unificou a teoria ao propor um modelo que explica o fluxo da informação no sistema metacognitivo (Veenman, HoutWolters, \& Afflerbach, 2006).

No modelo de Nelson e Narrens $(1990,1996)$ há uma clara tentativa de relacionar a cognição à metacognição, por meio do entendimento do fluxo da informação entre dois níveis, o nível meta e o objeto que são os níveis de atuação metacognitiva e cognitiva, respectivamente. Para os dois autores, o mecanismo de funcionamento do sistema metacognitivo permitiria que a cognição fluísse para o nível meta e para o nível objeto. No momento da passagem para o nível meta, ocorreria o monitoramento do nível objeto pela construção de um modelo que significaria a representação mental da realidade do processo cognitivo atual. Sendo assim, o monitoramento se caracteriza pelo acesso ou avaliação do progresso, ou estado atual, de um determinado empreendimento cognitivo. Por outro lado, quando a cognição flui para o nível objeto, ocorreria o controle por meio da regulação do processo cognitivo. Dessa maneira, em razão da informação advinda do nível meta, a cognição no nível objeto possui requisitos para se auto-regular, de acordo com as demandas da atividade cognitiva. Fica evidente, então, que o controle pode interromper uma atividade cognitiva, permitir sua continuidade e até modificá-la, criando, assim, uma hierarquia no sistema cognitivo, hierarquia essa que permite ao nível meta alterar o nível objeto. Assim, para que ocorram os processos de monitoramento e autorregulação, é essencial que haja fluxo de informação entre os dois níveis citados. Para o monitoramento, a informação deve fluir do nível objeto para o nível meta e para a autorregulação, a informação deve fluir do nível meta para o nível objeto (Nelson \& Narens, 1996).

Apesar das definições de controle e monitoramento metacognitivos serem distintas, os dois processos atuam de maneira conjunta (Boruchovitch, Schelini, \& Santos, 2010). Assim, o controle de alguma atividade cognitiva, como o entendimento de um texto lido em uma revista, depende do monitoramento da eficácia/ineficácia das estratégias utilizadas pelo indivíduo durante a leitura.

Jou e Sperb (2006) afirmam que, após a análise dos modelos de Flavell (1979) e Nelson e Narens (1990, 1996), é possível integrá-los, uma vez que Flavell focaliza os componentes ou as estruturas que compõem o sistema metacognitivo e Nelson e Narens, o processo de fluxo da informação desse mesmo sistema. Essa integração permite um melhor entendimento do funcionamento metacognitivo sob uma perspectiva mais ampla.

A metacognição é adquirida e desenvolvida por meio da experiência e do acúmulo de conhecimento e, graças a ela, os indivíduos são capazes de monitorar e regular seus desempenhos, bem como elaborar estratégias para potencializar a cognição. Sob esses aspectos nota-se a relevância do estudo e entendimento das habilidades metacognitivas direcionadas ao contexto educacional (Jou
\& Sperb, 2006). Flavell, Miller e Miller (1999) apontam para a importância do estudo da metacognição voltada para a educação, uma vez que estudantes capazes de monitorar, avaliar seus processos de aprendizagem e alterar as estratégias que estão utilizando, apresentam desempenho mais satisfatório nas tarefas escolares quando comparados aos alunos incapazes de realizar os mesmos procedimentos. Dados semelhantes a esses foram encontrados por Vadhan e Stander (1994), Boruchovitch (1999), Chahon (2006), Neves (2007) e Metcalf (2009). Além desses, outros autores também discorreram sobre metacognição aplicada à educação, entretanto, por se tratar do foco desta pesquisa apenas serão abordados nesse momento autores que utilizaram instrumentos para mensuração dessas habilidades em seus procedimentos.

$\mathrm{Na}$ literatura internacional é possível citar o Inventário de Conscientização Metacognitiva (MAI - Metacognitive Awareness Inventory), que avalia o conhecimento e a regulação cognitiva, formulado por Shraw e SperlingDenisson (citado por Panoura \& Philippou, 2005); os Questionários Metacognitivos (Meta-Cognitions Questionaires, $M C Q$ ), criados por Cartwright-Hatton e Well (1997) que relacionam alguns aspectos da metacognição ao desenvolvimento e à manutenção de desordens psicológicas como o transtorno de ansiedade generalizada; a versão reduzida do MCQ, o MCQ-30, (Wells \& Cartwright-Hatton, 2004); o Questionário de Lacuna na Mente (Blank in the Mind Questionnaire - BIMQ), elaborado por Moraitou e Efklides (2009), que considera o fenômeno de lacuna na mente como um fenômeno metacognitivo; o Inventário de Consciência Metacognitiva de Estratégias de Leitura (Metacognitive Awareness of Reading Strategies InventoryMARSI), formulado por Mokhtari e Reichard (2002), partindo do pressuposto de que consciência e monitoramento dos próprios processos de compreensão são aspectos críticos para a habilidade na leitura, entre outros instrumentos.

Já na literatura nacional, tem-se a Escala de Estratégias de Leitura - Formato Universitário (Joly, Cantalice, \& Vendramini, 2004), que avalia as estratégias de leitura dos indivíduos; a Escala de Estratégias de Leitura - Nível Fundamental I (Joly, 2006) e a Escala Metacognitiva de Leitura aplicada ao ensino médio - EMeL-EM (Joly, Santos, \& Marini, 2006), ambas derivadas da primeira escala; a Escala de Estratégias de Aprendizagem para Alunos do Ensino Fundamental (EAEF), formulada por Boruchovitch et al. (2006) e; a Escala de Avaliação das Estratégias de Aprendizagem para o Ensino Fundamental (EAVAP-EF), de autoria de Oliveira, Boruchovitch e Santos (2010), as duas mensurando as estratégias de aprendizagem dos alunos.

Do exposto, pode-se notar que a literatura nacional apresenta poucos relatos sobre instrumentos destinados à mensuração da metacognição, além do que, os instrumentos encontrados destinam-se a aspectos específicos do tema, como leitura e estratégias de aprendizagem para tarefas acadêmicas. Por essa razão, surge a oportunidade de elaboração de uma forma de avaliação que abranja não apenas habilidades relativas ao contexto acadêmico, mas também habilidades presentes em contextos gerais, por exemplo, quando se está assistindo televisão ou lendo um livro em sua casa. 
Dessa maneira, em estudo anterior (Pascualon-Araujo \& Schelini, 2013), foi apresentada a elaboração da Escala de Metacognição (EMETA), a análise da adequação dos itens e das instruções (evidência de validade baseada no conteúdo) e análise semântica possibilitada pela aplicação em amostra reduzida de participantes. Após algumas modificações sugeridas pelos especialistas e adequações advindas da aplicação em amostra reduzida, pretendeu-se com o presente estudo dar continuidade ao processo de análise da escala, avaliando as evidências de validade baseadas na estrutura interna por meio da análise fatorial (validade fatorial); a consistência interna (precisão) da escala, por meio do cálculo do coeficiente Alpha de Cronbach e a realização de análise de comparação entre as médias para investigar possíveis influências das variáveis gênero, tipo de escola e idade no desempenho dos participantes.

\section{Método}

\section{Participantes}

O estudo foi desenvolvido com 196 participantes, com idade entre 9 e 12 anos, 104 (53\%) do sexo masculino e $92(47 \%)$ do sexo feminino, sendo que $66 \%$ estavam matriculados em escolas públicas e $34 \%$ em escolas particulares, localizadas em uma cidade do interior do estado de São Paulo. Desses alunos, 27\% apresentavam 9 anos, $29 \%$ apresentavam 10 anos, 26\% apresentavam 11 anos e $18 \%$ apresentavam 12 anos. A distribuição dos participantes de acordo com as séries escolares foi a seguinte: $3 \%, 26 \%$, $51 \%$ e $20 \%$, respectivamente na $3^{\circ}$ série, $4^{\circ}$ série, $5^{\circ}$ série e $6^{\circ}$ série do Ensino Fundamental.

\section{Instrumento}

O instrumento utilizado foi a Escala de Metacognição (EMETA) formulada pelas autoras. A escala é do tipo Likert de seis pontos, sendo que o participante tem que escolher uma dentre seis possibilidades de resposta. As possibilidades de resposta são representadas ao mesmo tempo por números (entre 1 e 6) e círculos, que vão aumentando de acordo com a numeração, conforme demonstra a Figura 1.

\begin{tabular}{|l|l|l|l|l|l|}
\hline 1 & 2 & 3 & 4 & 5 & 6 \\
\hline & $\bullet$ & $\bullet$ & $\bullet$ & $\bullet$ & $\bigcirc$ \\
\hline
\end{tabular}

Figura 1. Possibilidades de resposta da Escala de Metacognição (EMETA) que variam de 1 (menor círculo) até 6 (maior círculo).

Se um participante considerasse que determinada afirmação da escala não era capaz de descrevê-lo ou representá-lo, ele deveria assinalar a primeira coluna, com o número um acompanhado pelo menor círculo. Se, ao contrário, acreditasse que o item era capaz de representá-lo completamente, assinalaria a última coluna, com o número seis acompanhado do maior círculo.
A EMETA foi formada por três exemplos fornecidos durante as instruções; quatro itens para o participante responder sozinho a fim de possibilitar a análise de seu entendimento da tarefa proposta; e pela escala propriamente dita, constituída por 67 itens divididos em duas subescalas: a subescala destinada à avaliação do conhecimento metacognitivo e a subescala para avaliação do controle ou auto-regulação cognitiva. As subescalas que compuseram a EMETA foram definidas baseando-se no relato apresentado por Ribeiro (2003) sobre os aspectos principais da metacognição serem, considerando-se o contexto educacional, o conhecimento metacognitivo (tomada de consciência dos procedimentos e das competências necessárias para a realização das tarefas) e o controle/autorregulação cognitivo (capacidade de avaliar a execução da tarefa e realizar adequações nos procedimentos utilizados quando necessário).

Alguns exemplos de itens do instrumento são: "Tento não conversar nas aulas que tenho mais dificuldades"; "Eu tento estudar um assunto aos poucos"; e, "Antes de começar uma tarefa, eu penso em muitos jeitos diferentes para resolvê-la".

\section{Procedimento}

O procedimento utilizado foi submetido e aprovado pelo Comitê de Ética em Pesquisa com seres humanos. Foi realizado contato com duas instituições de ensino, uma pública e outra privada, solicitando a autorização para a realização da coleta de dados com os alunos. Nesse contato inicial ocorreu uma breve apresentação da pesquisa, explicitação da sua relevância e contribuição para a educação, além da apresentação da escala. Após obtenção de autorização por parte das diretorias das instituições, o Termo de Consentimento Livre e Esclarecido (TCLE) foi encaminhado aos responsáveis pelos alunos selecionados por meio das educadoras de cada sala. Apenas alunos cujos pais autorizaram a participação constituíram a amostra desta pesquisa, que foi composta por participantes entre 9 e 12 anos de idade pertencentes a 12 salas diferentes distribuídos entre duas instituições de ensino.

A escala foi aplicada de forma coletiva na sala de aula dos participantes no mesmo período em que eles frequentavam a escola. Inicialmente, a pesquisadora responsável (uma das autoras) e duas auxiliares de pesquisa se apresentaram aos alunos, informando que diante de qualquer dúvida, eles deveriam levantar a mão e aguardar que uma delas iria atendê-los. Feito isso, o material foi entregue. A pesquisadora responsável disse que leria as instruções em voz alta e pediu para que eles a acompanhassem. Caso não tivessem entendido alguma palavra ou frase, eles deveriam circulá-las e, terminada a leitura, deveriam levantar a mão e aguardar pela ajuda. Sanadas as possíveis dúvidas, a experimentadora pediu para que os alunos respondessem aos itens da escala em silêncio.

Devido à necessidade de certa habilidade de leitura por parte dos participantes, visto que eles deveriam ler sem ajuda externa os itens da EMETA, foi solicitado à educadora de cada sala que informasse à pesquisadora responsável quais alunos apresentavam dificuldades de leitura. Esse procedimento foi adotado para excluir os participantes que, possivelmente, 
teriam dificuldade na leitura dos itens, gerando respostas imprecisas. As educadoras informaram a existência de quatro

Tabela1. Representação da carga fatorial para cada item da Escala de Metacognição (EMETA)

\begin{tabular}{|c|c|c|c|c|c|c|}
\hline Item & F1 & F2 & F3 & F4 & F5 & F6 \\
\hline 1 & & & 0,41 & & & \\
\hline 2 & & 0,29 & & & & \\
\hline 3 & & & 0,29 & & & \\
\hline 4 & 0,35 & & & & & \\
\hline 5 & & & & & 0,37 & \\
\hline 6 & 0,43 & & & & & \\
\hline 7 & 0,36 & & & & & \\
\hline 8 & 0,46 & & & & & \\
\hline 9 & 0,29 & & & & & \\
\hline 10 & 0,46 & & & & & \\
\hline 11 & 0,38 & & & & & \\
\hline 12 & 0,35 & & & & & \\
\hline 13 & 0,44 & & & & & \\
\hline 14 & & & & & & 0,36 \\
\hline 15 & & 0,37 & & & & \\
\hline 16 & & & 0,34 & & & \\
\hline 17 & 0,54 & & & & & \\
\hline 18 & 0,41 & & & & & \\
\hline 19 & 0,51 & & & & & \\
\hline 20 & 0,39 & & & & & \\
\hline 21 & 0,39 & & & & & \\
\hline 22 & 0,35 & & & & & \\
\hline 23 & 0,48 & & & & & \\
\hline 24 & 0,38 & & & & & \\
\hline 25 & 0,34 & & & & & \\
\hline 26 & 0,44 & & & & & \\
\hline 27 & 0,51 & & & & & \\
\hline 28 & 0,48 & & & & & \\
\hline 29 & & 0,45 & & & & \\
\hline 30 & 0,37 & & & & & \\
\hline 31 & & & 0,37 & & & \\
\hline 32 & 0,49 & & & & & \\
\hline 33 & 0,48 & & & & & \\
\hline 34 & 0,46 & & & & & \\
\hline 35 & 0,44 & & & & & \\
\hline 36 & & 0,37 & & & & \\
\hline 37 & 0,41 & & & & & \\
\hline 38 & 0,51 & & & & & \\
\hline 39 & 0,46 & & & & & \\
\hline 40 & 0,52 & & & & & \\
\hline 41 & 0,44 & & & & & \\
\hline
\end{tabular}

Tabela1. Representação da carga fatorial para cada item da Escala de Metacognição (EMETA) cont.

\begin{tabular}{|c|c|c|c|c|c|c|}
\hline Item & F1 & F2 & F3 & F4 & F5 & F6 \\
\hline 42 & & & & 0,33 & & \\
\hline 43 & & & 0,43 & & & \\
\hline 44 & & & 0,34 & & & \\
\hline 45 & 0,53 & & & & & \\
\hline 46 & 0,38 & & & & & \\
\hline 47 & 0,51 & & & & & \\
\hline 48 & 0,35 & & & & & \\
\hline 49 & & 0,46 & & & & \\
\hline 50 & & & & & 0,51 & \\
\hline 51 & 0,49 & & & & & \\
\hline 52 & 0,60 & & & & & \\
\hline 53 & 0,24 & & & & & \\
\hline 54 & 0,33 & & & & & \\
\hline 55 & 0,55 & & & & & \\
\hline 56 & & & & 0,49 & & \\
\hline 57 & 0,41 & & & & & \\
\hline 58 & 0,51 & & & & & \\
\hline 59 & 0,43 & & & & & \\
\hline 60 & 0,52 & & & & & \\
\hline 61 & 0,45 & & & & & \\
\hline 62 & 0,49 & & & & & \\
\hline 63 & 0,45 & & & & & \\
\hline 64 & 0,44 & & & & & \\
\hline 65 & 0,46 & & & & & \\
\hline 66 & 0,47 & & & & & \\
\hline 67 & 0,35 & & & & & \\
\hline
\end{tabular}

Nota.Os sinais indicando cargas negativas nos itens foram eliminados, pois não foram considerados na análise.

F1: fator 1; F2: fator 2; F3: fator 3; F4: fator 4; F5: fator 5 e F6: fator 6 .

participantes que apresentavam dificuldades de leitura que os impediriam de responder à escala adequadamente. A eles foi entregue a escala como aos outros, porém, no momento da entrega, esses instrumentos foram excluídos.

\section{Resultados}

A análise fatorial exploratória revelou a existência de seis fatores que explicam $34,11 \%$ da variância total da escala, sendo $16,84 \%$ determinados pelo fator $1 ; 4,82 \%$ determinados pelo fator $2 ; 3,34 \%$ determinados pelo fator $3 ; 3,17 \%$ determinados pelo fator $4 ; 3,03 \%$ determinados pelo fator 5 ; e 2,91\% determinados pelo fator 6 . Observa-se que o fator 1 explica $16,84 \%$ da variância total da escala equivalendo, aproximadamente, à soma da variância dos outros cinco fatores da EMETA, que alcança 17,27\%. Sendo 
assim, o fator 1 explica a maior parte da variância total do instrumento, cerca de 50\%.

A análise das cargas fatoriais dos itens da Escala de Metacognição relevou que quatro deles apresentaram valores abaixo de 0,30. A partir do critério utilizado por Kline (1994), no qual os valores abaixo de 0,30 tendem a responder por menos de $9 \%$ da variância total do instrumento, os quatro itens foram excluídos.

A divisão dos itens entre os seis fatores foi realizada comparando-se os valores de suas cargas nos fatores. $\mathrm{O}$ item foi considerado como pertencente a um fator quando apresentou uma carga mais elevada neste fator, desprezandose o sinal, uma vez que este apenas ilustra se a relação é negativa ou positiva. Nenhum item apresentou carga em dois ou mais fatores simultaneamente, todos carregaram apenas em um fator.

Após distribuição dos 63 itens, o fator 1 ficou constituído por 49 itens, o fator 2 ficou constituído por quatro itens, o fator 3 ficou constituído por quatro itens, o fator 4 ficou constituído por três itens, o fator 5 ficou constituído por dois itens e o fator 6 ficou constituído por um item.

$\mathrm{Na}$ análise da composição dos itens nos fatores, estabeleceu-se que o número mínimo de itens para se considerar o fator como relevante para a escala seria cinco, uma vez que um fator formado por poucos itens, provavelmente, não teria utilidade para fins de avaliação, pelo fato de poder não ser representativo da amostra de comportamento relacionada ao fator em questão. Desta forma, os fatores 2, 3, 4, 5 e 6, bem como os itens que os constituíam foram eliminados da Escala de Metacognição (EMETA), pois não atingiram este número mínimo. Portanto, a nova versão da EMETA ficou constituída por apenas um fator que explica $16,84 \%$ da variância total.

Os coeficientes Alpha de Cronbach dos itens da EMETA encontrados foram elevados, situando-se entre 0,917 e 0,919 . Apesar de testes da significância estatística para o Alpha não serem disponíveis em sistemas computacionais, o valor encontrado foi excelente, uma vez que é consenso que o limite inferior aceitável para ele é de 0,70 ou ainda 0,60 para pesquisas exploratórias (Fachel \& Camey, 2000).

Após observação do conjunto de 49 itens da EMETA, verificou-se a existência de itens semelhantes entre si e, pelo fato dessa semelhança poder ser prejudicial à aplicação do instrumento, uma vez que os participantes poderiam considerar as afirmações repetitivas, realizouse o agrupamento dos itens semelhantes para permitir a exclusão de alguns deles. A semelhança considerada para o agrupamento se baseou nas habilidades abordadas por eles, por exemplo, monitoramento após leitura de livro e estratégias para compreender melhor uma tarefa que the foi ensinada. Foram formados 11 agrupamentos desse tipo. O primeiro critério utilizado para escolha dos itens que permaneceriam na EMETA foi a comparação das cargas fatoriais dentro de cada grupo, eliminando-se itens com valores mais baixos em comparação com seus semelhantes. O segundo critério foi a compreensão do item, excluindo-se todos os que poderiam apresentar dificuldades de compreensão pelos participantes durante a aplicação. $\mathrm{O}$ valor do Alpha de Cronbach não foi utilizado como critério de exclusão, uma vez que todos os itens tinham valores acima de 0,90 .
Os itens 11 ("Em uma brincadeira sempre penso se estou indo bem ou não."), 30 ("Eu penso em várias maneiras para solucionar um problema e escolho a melhor"), 38 ("Quando estou fazendo uma tarefa, às vezes eu paro para ver se estou entendendo"), 39 ("Eu sei quando entendi a história de um livro") e 66 ("Quando eu leio um livro paro frequentemente para ver se eu entendi o que já li") foram eliminados devido às suas baixas cargas fatoriais. Já os itens 6 ("Eu posso tentar encontrar a vontade de aprender quando eu preciso"), 17 ("Eu presto atenção em como minha mente funciona"), 48 ("Eu presto atenção no problema como um todo e não nos detalhes dele") e 55 ("Pensando na minha inteligência, uso meus pontos fortes para compensar os fracos"), foram eliminados em razão de serem itens constituídos por conceitos pouco familiares aos participantes e que, possivelmente, dificultariam a compreensão dos itens, como "vontade de aprender", "funcionamento da mente", "problema como um todo" e "minha inteligência". Deste modo, foram excluídos nove itens da Escala de Metacognição que passou para 40 em sua configuração final.

Realizou-se, no único fator, uma análise para verificar a consistência interna (precisão) dos seus itens por meio do cálculo do coeficiente Alpha de Cronbach que, segundo Pasquali (2003), é também uma forma de avaliar a validade de construto de um instrumento psicológico. $\mathrm{O}$ resultado encontrado foi 0,90, índice considerado elevado de acordo com Murphy e Davidshofer (1998). Para esses autores, valores em torno de 0,80 são considerados moderados a altos, valores em torno de 0,70 são considerados baixos e valores iguais ou inferiores a 0,60 são inaceitavelmente baixos. Para Guay, Boggiano e Vallerand (2001), o valor obtido pela EMETA também é considerado alto, uma vez que eles consideram como aceitáveis para escalas de autorrelato, valores de consistência interna entre 0,70 e 0,80 .

A análise da consistência interna dos 40 itens da EMETA revelou que todos os valores de correlação com o total são adequados a uma escala, pois foram superiores a 0,30 (Kline, 1994). Em razão disso, a exclusão de qualquer item do instrumento não geraria significativas alterações no Coeficiente Alpha de Cronbach, permanecendo sempre em torno de 0,89 .

Foi realizada também análise de comparação entre as médias para investigar a influência das variáveis gênero, tipo de escola e idade dos participantes. Interessante comentar nesse momento que as respostas da escala apresentaram distribuição normal e, devido a isso, podese afirmar que o conjunto de itens analisado proporciona melhor discriminação entre as extensões das magnitudes do construto medido. A Tabela 2 indica os resultados da análise de variância (ANOVA) para a variável gênero.

Tabela 2. Análise de variância (ANOVA) para a variável gênero

\begin{tabular}{lcccc}
\hline ANOVA & $\begin{array}{c}\text { Graus de } \\
\text { Liberdade } \\
(\mathrm{df})\end{array}$ & $\begin{array}{c}\text { Soma dos } \\
\text { quadrados }\end{array}$ & $F$ & $p$ \\
\hline Modelo & 1 & 581,12 & 0,56 & 0,4560 \\
Erro & 194 & 202071,63 & & \\
TOTAL & 195 & 202652,75 & & \\
\hline
\end{tabular}


Para essa variável, a ANOVA não indicou que as médias das respostas apresentaram diferença significativa entre participantes do gênero masculino e feminino $(F(1,194)=$ $0,56 ; p=0,4560 ; \alpha=0,05)$. De acordo com esses resultados, a pontuação atingida pelos participantes na Escala de Metacognição não depende do seu gênero, isto é, a variável gênero não produz efeito sobre as respostas apresentadas pelos participantes na escala. A Tabela 3 apresenta os dados para a variável tipo de escola.

Em relação à variável tipo de escola, a $A N O V A$ demonstrou que não há diferença significativa entre as médias das respostas dos participantes matriculados em escolas públicas e escolas privadas $(F(1,194)=0,79 ; p=0,3764$; $\alpha=0,05)$ como representado na Tabela 3. Desse modo, é possível afirmar que o desempenho dos participantes na Escala de Metacognição independe do tipo de escola que frequenta, ou seja, as respostas de alunos matriculados em escolas públicas e alunos matriculados em escolas privadas não dependem do tipo de escola frequentada.

Tabela 3. Análise de variância (ANOVA) para a variável Escola

\begin{tabular}{lcccc}
\hline ANOVA & $\begin{array}{c}\text { Graus de } \\
\text { Liberdade } \\
\text { (df) }\end{array}$ & $\begin{array}{c}\text { Soma dos } \\
\text { quadrados }\end{array}$ & $F$ & $p$ \\
\hline Modelo & 1 & 817,8556 & 0,79 & 0,3764 \\
Erro & 194 & 201834,8944 & & \\
TOTAL & 195 & 202652,7500 & & \\
\hline
\end{tabular}

A Tabela 4 representa a análise da variável idade. A variável idade também não apresentou diferença significativa entre as médias das respostas dos participantes segundo a $\operatorname{ANOVA}(F(3,192)=1,98 ; p=0,1190 ; \alpha=0,05)$, indicando que as respostas dos alunos na Escala de Metacognição independem de suas idades.

Por meio das informações relatadas nessa seção, observou-se que as avaliações acerca das evidências de

Tabela 4. Análise de variância (ANOVA) para a variável Idade

\begin{tabular}{lcccc}
\hline ANOVA & $\begin{array}{c}\text { Graus de } \\
\text { Liberdade } \\
\text { (df) }\end{array}$ & $\begin{array}{c}\text { Soma dos } \\
\text { quadrados }\end{array}$ & $F$ & $p$ \\
\hline Modelo & 3 & 6069,4354 & 1,98 & 0,1190 \\
Erro & 192 & 196583,3146 & & \\
TOTAL & 195 & 202652,7500 & & \\
\hline
\end{tabular}

validade e precisão da Escala de Metacognição apresentaram resultados satisfatórios.

\section{Discussão}

Por meio dos resultados apresentados pode-se dizer que os objetivos iniciais de elaborar uma escala direcionada a mensurar a metacognição infantil, bem como analisar algumas de suas propriedades psicométricas, foram alcançados por meio do estudo realizado. $\mathrm{O}$ processo de elaboração da escala procurou ir ao encontro da situação atual da área de avaliação psicológica no Brasil, acordando com a resolução $n^{\circ}$. 02/2003 do Conselho Federal de Psicologia que, dentre outros aspectos, define diretrizes para a elaboração, utilização e realização de estudos para verificar as evidências de validade e precisão de instrumentos. Desta maneira, este estudo tenta contribuir com a evolução da área de avaliação psicológica no país e com a literatura sobre metacognição, uma vez que não foi encontrado instrumento nacional que atue como uma espécie de rastreio das habilidades metacognitivas como proposto pela Escala de Metacognição.

Para a construção do instrumento, devido à amplitude da definição de metacognição encontrada na literatura (Cavanaugh \& Perlmutter, 1982; Dunlosky \& Metcalfe, 2009; Flavell, 1979; Vadhan \& Stander, 1994; Veenmam, Van Hout-Wolters, \& Afflerbach, 2006), adotou-se, neste estudo, a definição exposta por Ribeiro (2003), que entende o conceito como formado por dois elementos básicos: o conhecimento metacognitivo e o controle ou autorregulação cognitivos. Como a formulação dos itens da EMETA foi baseada nessas duas amplitudes, era esperado que elas constituíssem dois fatores, que responderiam pela maior porcentagem de variância da escala. Entretanto, esse fato não foi observado após realização da análise fatorial exploratória que apontou a existência de seis fatores. Possivelmente, a elaboração dos itens não contemplou apenas as duas dimensões propostas por Ribeiro (2003).

É provável que a configuração final da EMETA, constituída por um único fator, seja reflexo da dependência das dimensões conhecimento metacognitivo e autorregulação ou monitoramento cognitivo, utilizadas como base para a elaboração dos itens. Apesar da existência dessa dependência entre as duas dimensões, reconhece-se que elas envolvem habilidades cognitivas diferentes, como a tomada de consciência no primeiro caso e o planejamento, supervisão e avaliação, no segundo.

Como observado por diferentes autores (Flavell, 1987; Ribeiro, 2003), as dimensões do conceito metacognição são intrinsecamente relacionadas, sendo uma dependente da outra para a sua existência. Assim, pode-se entender que seria difícil um indivíduo regular suas atividades cognitivas (autorregulação ou monitoramento cognitivos) sem ter conhecimento (conhecimento metacognitivo) de quais são as habilidades metacognitivas que ele possui em seu repertório. Dessa forma, o resultado encontrado neste estudo fornece mais uma evidência para fortalecer a noção apontada por outros autores acerca da dependência entre as dimensões conhecimento metacognitivo e autorregulação cognitiva.

A retirada dos itens compreendidos nos fatores 2, 3, 4, 5 e 6 pouco prejudicou a representatividade do conceito mensurado pela escala, uma vez que observou-se que os itens que compunham esses fatores abrangeram aspectos semelhantes aos abrangidos pelo único fator que continha mais de cinco itens, assegurando que nenhuma dimensão abordada por esses itens eliminados seria excluída do instrumento.

A versão final da Escala de Metacognição ficou composta por itens que estão relacionados a aspectos como: utilização de estratégias para avaliar o próprio desempenho durante e após a execução de uma tarefa, mudança de estratégia ineficiente adotada em uma atividade, conhecimentos 
relativos às tarefas a serem realizadas, conhecimento acerca de características pessoais e conhecimento sobre variáveis relacionadas a desempenhos mais eficientes em tarefas específicas. Por abranger características gerais do conceito metacognição, o único fator existente na escala foi nomeado como "Metacognição". Por fator único entende-se que os diferentes aspectos da metacognição são avaliados por itens que apresentam um conceito em comum, fato esse que determina a não existência de dois ou mais fatores que abrangeriam aspectos diferentes do conceito.

Durante a aplicação na amostra ampla, não foram encontradas dificuldades significativas relacionadas ao entendimento da tarefa proposta e dos itens listados, as poucas dúvidas que surgiram foram respondidas individualmente para cada participante. Como se esperava, alguns participantes demonstraram desinteresse em responder aos itens finais da EMETA, possivelmente devido à sua extensão inicial de 67 itens. Porém, pensa-se ter diminuído essa dificuldade, uma vez que após análise de dados e estabelecimento do fator único Metacognição, o número de itens caiu para 40 , aproximadamente $60 \%$ da extensão inicial.

Além das análises já descritas, foram realizadas também análises de comparações entre as médias dos participantes para as variáveis gênero, tipo de escola e idade. Diferentemente dos dados obtidos na análise da EMETA, a literatura apresenta alguns relatos de melhor desempenho nas tarefas propostas por parte de pessoas do sexo feminino quando comparadas a pessoas do sexo masculino. Joly (2006), estudando o uso de estratégias metacognitivas direcionadas à leitura em participantes do ensino fundamental, encontrou maior utilização dessas estratégias entre as meninas. Focalizando as mesmas habilidades, mas dessa vez em alunos do ensino médio, Joly, Santos e Marini. (2006) também encontraram dados que concordaram com os referentes aos alunos do ensino fundamental. Um ponto interessante acerca desse resultado discordante deve ser apresentado neste momento. Os dados relacionando habilidades metacognitivas e outras características dos indivíduos, no caso o gênero dos participantes, são provenientes de estudos realizados com população diferente da população focalizada neste texto, o que pode explicar, em partes, a diferença encontrada no desempenho entre meninos e meninas. Dessa maneira, devese considerar, para as análises realizadas, a possibilidade de que as habilidades avaliadas na amostra utilizada neste trabalho se apresentem de maneira não semelhante às habilidades avaliadas em outras populações. Para se obter uma comparação mais fidedigna, é necessária a avaliação das mesmas habilidades na mesma população, algo que a literatura ainda não aborda.

Observando-se a variável tipo de escola Joly et al. (2006) apresentaram resultados que vão ao encontro dos obtidos para a Escala de Metacognição.

Em relação à idade dos participantes, o resultado obtido com a aplicação da EMETA também foi encontrado por Joly (2006) em relação à utilização de estratégias metacognitivas de leitura em participantes do ensino fundamental. Entretanto, Joly et al. (2006), investigando participantes do ensino médio, encontraram diferença significativa quanto ao uso de estratégias metacognitivas de leitura entre 15 e 17 anos de idade. Moraitou e Efklides (2009) também encontraram influência da variável idade analisando os desempenhos de seus participantes no fator falta de conhecimento (lack of knowledge) no Questionário de Lacuna na Mente ("The Blank in the Mind Questionnaire").

Cabe comentar que, além disso, os dados observados na EMETA não concordam com o exposto por autores como Flavell (1979), Flavell et al. (1999); Flavell e Wellman (1975), Ribeiro (2003), Neves (2007), Veenmam et al. (2006) e Vygotsky (1978), quando comentam sobre o desenvolvimento de habilidades metacognitivas. Segundo esses autores, essas habilidades evoluem de acordo com o desenvolvimento cognitivo dos indivíduos e, sendo assim, indivíduos com idades mais elevadas se desempenhariam melhor do que indivíduos de idades menos elevadas, quando comparados em tarefas iguais.

Algumas hipóteses acerca desse resultado foram levantadas. Uma delas está relacionada ao próprio desenvolvimento das habilidades metacognitivas. Apesar dos relatos científicos (Flavell, 1979; Veenmam et al., 2006) apresentarem 8 anos como sendo a idade em que essas habilidades surgem no repertório dos indivíduos, os dados encontrados no presente estudo direcionam para uma análise mais minuciosa dos itens, uma vez que não se encontrou diferença significativa no desempenho dos participantes das quatro faixas etárias abordadas na amostra, que pode ser devido ao fato de os itens não contemplarem a diferença entre os aspectos do conhecimento metacognitivo e da autorregulação cognitiva, como se pretendeu no momento da elaboração dos mesmos.

Outra hipótese é que pode-se supor que essas duas habilidades se desenvolvam lentamente nos primeiros anos (entre 9 e 12 anos de idade) de maneira a não demonstrar diferenças significativas entre os desempenhos dos indivíduos e, posteriormente a essa idade, o desenvolvimento seja mais rápido e as diferenças encontradas entre os desempenhos dos indivíduos sejam mais evidentes.

Além dessa hipótese do desenvolvimento, outra hipótese levantada diz respeito à possibilidade das habilidades metacognitivas envolvidas no conhecimento metacognitivo e na autorregulação mensuradas pela escala não se apresentarem distintas na faixa etária abrangida no estudo, isto é, as habilidades estão relacionadas entre si de maneira tão intrínseca que dificulta a separação em duas classes de habilidades diferentes. Talvez essa separação ocorresse em uma idade mais avançada, como em estudantes do ensino médio e universitários, como mostra a literatura.

Essa não concordância também implicou o descarte de uma fonte de evidências de validade, a chamada evidência com base na relação com variáveis externas. Neste estudo, a variável externa era a idade cronológica do participante que, segundo a literatura, seria um preditor de desempenho em tarefas envolvendo habilidades metacognitivas, uma vez que era esperado que indivíduos com mais idade se desempenhassem melhor do que indivíduos com menos idade.

Uma consideração acerca da análise das comparações entre as médias deve ser feita. Em razão de não terem sido encontrados na literatura instrumentos que mensurassem as habilidades metacognitivas de maneira mais geral, como realizado pela EMETA, os dados do presente estudo 
foram discutidos à luz de pesquisas utilizando instrumentos nacionais e internacionais que abordaram habilidades metacognitivas específicas, como estratégias direcionadas à leitura e resolução de problemas matemáticos.

Além disso, a população-alvo utilizada nos estudos envolvendo a mensuração de habilidades metacognitivas foi em sua maioria estudantes universitários e, é possível que os resultados encontrados para essa população sejam diferentes dos resultados encontrados para uma população com idade inferior, como a utilizada neste estudo (participantes entre 9 e 12 anos de idade). Evidencia-se nesse momento a contribuição que o presente estudo proporciona para a área de desenvolvimento da metacognição, uma vez que investiga o repertório de habilidades metacognitivas em uma população pouco abordada na literatura atual.

Após os resultados serem analisados e os objetivos da pesquisa contemplados de forma satisfatória, acredita-se que o texto apresentado tenha contribuído para o campo de estudos sobre a metacognição, principalmente para a área de avaliação. Apesar disso, reconhece-se que esse foi apenas um passo diante de uma ampla gama de aspectos ainda a serem estudados sobre o conceito. Particularmente, a realização de novos estudos com o objetivo de investigar outros parâmetros psicométricos da Escala de Metacognição se faz necessário para torná-la um instrumento válido para a mensuração de habilidades metacognitivas em participantes entre 9 e 12 anos de idade.

No futuro, após estudos adicionais de validade e do estabelecimento de normas, a aplicação da EMETA poderá ser considerada um norte para observações mais atentas daqueles participantes que apresentem resultados abaixo do esperado.

\section{Referências}

Boruchovitch, E. (1999). Estratégias de aprendizagem e desempenho escolar: considerações para a prática educacional. Psicologia: Reflexão e Crítica, 12(2), 361-376.

Boruchovitch, E., Santos, A. A. A., Costa, E. R., Neves, E. R. C., Cruvinel, M., Primi, R., \& Guimarães, S. E. R. (2006). A construção de uma escala de estratégias de aprendizagem para alunos do ensino fundamental. Psicologia: Reflexão e Crítica, 22(3), 297-304.

Boruchovitch, E., Schelini, P. W., \& Santos, A. A. A. (2010). A metacognição: conceituação e medidas. In A. A. A. dos Santos, F. F. Sisto, E. Boruchovitch \& E. Nascimento (Eds.), Perspectivas em avaliação psicológica (pp. 123-143). São Paulo: Casa do Psicólogo.

Cartwriht-Hatton, S., \& Wells, A. (1997). Beliefs about worry and intrusions: the meta-cognitions questionnaire and its correlates. Journal of Anxiety Disorders, 11(3), 279-296.

Cavanaugh, J. C., \& Perlmutter, M. (1982). Metamemory: a critical examination. Child Development, 53, 11-28.

Chahon, M. (2006). Metacognição e resolução de problemas aritméticos verbais: teoria e implicações pedagógicas. Revista do Departamento de Psicologia - UFF, 2, 163-176.

Conselho Federal de Psicologia - CFP. (2003). Resolução no. 002/2003. Recuperado de http://www.pol.org.br.
Dunlosky, J., \& Metcalf, J. (2009). Metacognition. Thousands Oak, CA: SAGE.

Fachel, J. M. G., \& Camey, S. (2000). Avaliação psicométrica: a qualidade das medidas e o entendimento dos dados. In J. A. Cunha (Ed.), Psicodiagnóstico V (pp. 158-170). Porto Alegre: Artes Médicas.

Flavell, J. H., Friedrichs, A. G., \& Hoyt, J. D. (1970). Developmental changes in memorization processes. Cognitive Psychology, 1, 324-340.

Flavell, J. H., \& Wellman, H. M. (1975). Metamemory. Washington, DC: National Science Foundation.

Flavell, J. H. (1976). Metacognitive aspects of problem solving. In L. B. Resnik (Ed.), The Nature of Intelligence (pp. 231-236). Hillsdale: Lawrence Erlbaum.

Flavel, J. H. (1979). Metacognition and Cognitive Monitoring: A new area of cognitive- developmental inquiry. American Psychologist, 34(10), 906-911.

Flavel, J. H. (1987). Speculations about the nature and development of metacognition. In F. Weinert \& R. Kluwe (Eds.), Metacognition, Motivation and Understanding (pp. 21-29). Hillsdale, NJ: Lawrence Erlbaum.

Flavell, J. H., Miller, P. H., \& Miller, S. A. (1999). Desenvolvimento Cognitivo (3 ed.). Porto Alegre: Artmed.

Guay, F., Boggiano, A. K., \& Vallerand, R. J. (2001). Autonomy support, intrinsic motivation and perceived competence: Conceptual and empirical linkages. Personality and Social Psychology Bulletin, 27, 643-650.

Joly, M. C. R. A. (2006). Escala de estratégias de leitura para a etapa inicial do ensino fundamental. Estudos em Psicologia Campinas, 23(3), 271-278.

Joly, M. C. R. A., Cantalice, L. M., \& Vendramini, C. M. M. (2004). Evidências de validade de uma escala de estratégias de leitura para universitários. Interação em Psicologia, 8(2), 261-270.

Joly, M. C. R. A., Santos, L. M., \& Marini, J. A. S. (2006). Uso de estratégias metacognitivas de leitura por alunos do ensino médio. Paidéia, 16(34), 205-212.

Jou, G. I., \& Sperb, T. M. (2006). A metacognição como estratégia reguladora da aprendizagem. Psicologia: Reflexão e Crítica, 19(2), 177-185.

Kline, P. (1994). An easy guide to factor analysis. New York: Routlege.

Metcalf, J. (2009). Metacognitive judgments and control of study. Current Directions in Psychological Science, 18(3), 159-163.

Mokhtari, K., \& Reichard, C. A. (2002). Assessing students' metacognitive awareness of reading strategies. Journal of Educational Psychology, 94(2), 249-259.

Moraitou, D., \& Efklides, A. (2009). The blank in the mind questionnaire (BIMQ). European Journal of Psychological Assessment, 25(2), 115-122.

Murphy, K. R., \& Davidshofer, C. O. (1998). Psychological testing: Principles and applications. Upper Saddle River: Prentice-Hall.

Nelson, T., \& Narens, L. (1990). Metamemory: A theoretical framework and new findings. The Psychology of Learning and Motivation, 26, 125-173.

Nelson, T., \& Narens, L. (1996). Why investigate metacognition? In J. Metclafe \& A. P. Shimamura (Eds.), Metacognition, Knowing about Knowing (pp. 1-27). Cambridge, MA: MIT Press. 
Neves, D. A. B. (2007). Leitura e metacognição: Uma experiência em sala de aula. Encontros Bibli: Revista Eletrônica de Biblioteconomia e Ciência da Informação, 24, 1-9. Retrieved from https://periodicos.ufsc.br/index.php/eb/article/ download/1518.../405

Oliveira, K. L., Boruchovitch, E., \& Santos, A. A. A. (2010). EAVAPEF - Escala de avaliação das estratégias de aprendizagem para o ensino fundamental. São Paulo: Casa do Psicólogo.

Panaoura, A., \& Philippou. G. (2005). The measurement of young pupils' metacognitive ability in mathematics: The case of selfrepresentation and self evaluation. CERME 4. Retrieved from http://cerme4.crm.es/Pa .../panaoura.philippou.pdf

Pascualon-Araujo, J. F, \& Schelini, P. W. (2013). Escala de Avaliação da Metacognição Infantil: evidências de validade e análise semântica. Avaliação Psicológica, 12(2), 147-156.
Pasquali, L. (2003). Psicometria - Teoria dos testes na Psicologia e na Educação (2a ed.), Petrópolis: Editora Vozes.

Ribeiro, C. (2003). Metacognição: um apoio ao processo de aprendizagem. Psicologia: Reflexão e Crítica, 16(1), 109-116.

Vadhan, V., \& Stander, P. (1994). Metacognitive ability and test performance among college students. The Journal of Psychology, 128(3), 307-309.

Veenman, M. V. J., Van Hout-Wolters, B. H. A. M., \& Afflerbach, P. (2006). Metacognition and learning: conceptual and methodological considerations. Metacognition and learning, 1, 3-14.

Vygotsky, L. S. (1978). Mind in Society. The Developmental of Higher Psychological Processes. Cambridge MA: Harvard University Press

Wells, A., \& Cartwright-Hatton, S. (2004). A short form of the metacognitions questionnaire: properties of the MCQ-30. Behaviour Research and Therapy, 42, 385-396.

Recebido em 16.08.2014

Primeira decisão editorial em 27.08.2014

Versão final em 16.10.2014

Aceito em 02.12.2014 